\title{
Effect of Mechanical Stretching of the Skin on Collagen Fibril Thermal Stability
}

\author{
*1El-ta'alu Abbas Bubakar, ${ }^{2}$ Alhassan Adamu Jibrin and ${ }^{1} \mathrm{lbrahim}$ Sulayman \\ 1Department of Human Physiology, Faculty of Medicine, Bayero University, Kano, Nigeria \\ ${ }^{2}$ Department of Biochemistry, Faculty of Science, Bayero University, Kano, Nigeria \\ [ ${ }^{\star}$ Corresponding Author: E-mail; abbaseltaalu@yahoo.co.uk]
}

\begin{abstract}
Stabilization of collagen fibres during development and through growth to maturation has now become fairly documented. In vitro effect of mechanical stretching of rats' skin on oxidative deamination of $\varepsilon$ $\mathrm{NH}_{2}$-groups of lysine and hydroxylysine, and functional properties of its type I collagen were studied. Experiments were carried out on the skin of a 3-months old male Wistar rat weighing about $800 \mathrm{mg}$. The skin tissue was divided into four (4) and subjected to different mechanical stresses in Ringer-Kreb's medium for 6 hours at $37^{\circ} \mathrm{C}$. The Degree of oxidative deamination of lysine and hydroxylysine was evaluated by way of the content of free $\varepsilon$-amino $\left(\varepsilon-\mathrm{NH}_{2}\right)$ and aldehyde $(\mathrm{COH})$ groups. Level of covalent cross-linking of collagen in skin samples was assessed by its solubility in $1 \mathrm{M}$ solution of $\mathrm{NaCl}$ and expressed as a percentage of total collagen content in the tissue. Collagen synthesized under the influence of mechanical stretching had higher content of free $\varepsilon-\mathrm{NH}_{2}$-groups and lower level of inter-molecular cross-links compared to the collagen synthesized without the stretching. This finding may indicate that fibrils formed in the former were shorter and had lower thermal stability due to a decrease in the degree of cross linkages.
\end{abstract}

Keywords: Connective tissue, collagen, cross-linkage, fibril, mechanical stretching, oxidative deamination, thermal stability.

\section{INTRODUCTION}

The overall shape and function of the body depends on the basic framework of collagen polymers that are stabilized by inter-molecular cross-links (Hyun et al., 2006). Stabilization of collagen molecule is a carefully controlled enzymatic process, which produces inter-molecular cross-links at specific locations (Hyun et al., 2006). Self-assembly of fibrillar collagen molecules in the sub-molecular aggregates is one of the most important stages of early morphogenesis of the connective tissue, which takes place in different processes - embryonic development, growth, periodic physiological modifications of organs, wound healing, and physical training (Hyun et al., 2006). As a result, fibres are virtually inextensible and provide exceptional tensile strength and resistance to mechanical forces. Deformation of cells due to mechanical stretching is a common event that takes place in the body, and it primarily applies to all varieties of the connective tissue, where mechanical loads are extremely diverse (Hyun et al., 2006), which may be permanent or cyclical in intensity and duration.

It was recently established that, deformation of connective tissue cells under the influence of mechanical stretching intensifies the synthesis of structural biopolymers, particularly those of the collagen molecules, which are capable of associating into fibrils by self assembly (Buschmann et al., 1995, Garbuzenko et al., 1997; Jin et al., 2001; Hyun et al.,
2006). There is a mismatch between the intensity of the assembly of collagen polypeptide chains on ribosomes, and the level of their post-translational modification, which is carried out by enzymes of processing (Kot et al., 2004; Persky et al., 2006). This effect may lead to a change in the final structure of the collagen protein, and hence, the properties of its molecules (Stepanova et al., 1996) showed that, the degree of proline hydroxylation, and accordingly, the thermal stability of type I collagen synthesized in vitro in the stretched skin is lower than that of collagen synthesized without mechanical stretching.

The most important step in the post-translational modification of collagen is the oxidative deamination of $\varepsilon-\mathrm{NH}_{2}$-groups of lysine and hydroxylysine under the action of lysyloxidase, with the transformation of these amine groups into allysyne and hydroxyallysyne, which contain aldehyde groups $(\mathrm{COH}-)$ in place of $\varepsilon-\mathrm{NH}_{2}$-groups (Sun et al., 2006). This modification is necessary for the formation of cross-covalent bonds in the submolecular collagen structures. These cross-covalent linkages are formed by the interaction of either two $\mathrm{COH}$-groups (internal aldole links), or $\varepsilon-\mathrm{NH}_{2}$-groups with $\mathrm{COH}$-groups (Schiff's bases) of adjacent molecules (Eyre and Wu, 2005). This study was initiated to, therefore, examine the relationship between the degree of oxidative deamination of $\varepsilon$ $\mathrm{NH}_{2}$-groups of lysine and hydroxylysine in type I collagen, synthesized in the skin under the influence 
of mechanical stretching, morphology, and thermal stability of sub-molecular structures.

At present, however, there are practically no reports on the relationship between the influence of mechanical stress of the skin and disruption of the formation of fibrillar collagen structures. It is therefore, expedient to deeply study the aforementioned relationship as well as the consequential decrease in the thermal stability of fibrils that are formed from collagen structures.

To achieve the aforementioned aim, the following objectives were set:

i. Estimate the content of de novo synthesized collagen.

ii. Determine the concentration of free $\varepsilon-\mathrm{NH}_{2-}$ groups of lysine and hydroxylysine.

iii. Determine the concentration of free aldehyde groups of allysine and hydroxyallysine.

iv. Examine the histology of skin samples.

v. Measure the thermodynamic parameters of denaturation of type I collagen.

The experimental animals were handled in accordance with international principles of the European Convention on protection of vertebrate animals used for experimental and other scientific purposes (European Convention, 1985), and norms of biomedical ethics in accordance with the Law of Ukraine on protecting animals from man-handling were observed in the experiments.

Animals were lulled to sleep by intravenous injection of sodium thiopental (Greene, 2002), and then decapitated. Skin samples $(20 \times 5) \mathrm{mm}$ were cleaned from subcutaneous fat and hair.

\section{Research Design}

The skin tissue was divided into four (4) and subjected to different mechanical stresses $(0.000$, $0.075,0.120,0.240 \mathrm{MN} / \mathrm{m}^{2}$ ) in Ringer-Kreb's medium for 6 hours, at $37^{\circ} \mathrm{C}$.

\section{MATERIALS AND METHODS} In vitro Skin Incubation

Samples of tissue were incubated in Ringer-Kreb's medium (composition: $9.075 \mathrm{~g}$ of tris $\mathrm{HCl} ; 8.2998 \mathrm{~g}$ $\mathrm{NaCl} ; 0.5206$ g KC1; $2222 \mathrm{~g} \mathrm{MgCl}_{2} ; 0.4333 \mathrm{~g}$ $\mathrm{K}_{2} \mathrm{HSO}_{4}$ and $5.4 \mathrm{~g}$ of $\mathrm{C}_{6} \mathrm{H}_{2} \mathrm{O}_{6}$ in 1 litre of distilled water) for 6 hours, at $37^{\circ} \mathrm{C}$ stretching the samples in the longitudinal direction with static stresses of $\sigma=$ $0.00 ; 0.075 ; 0.120$ and $0.240 \mathrm{MN} / \mathrm{m}^{2}$ Mechanical stresses applied to the samples were calculated using the formula:

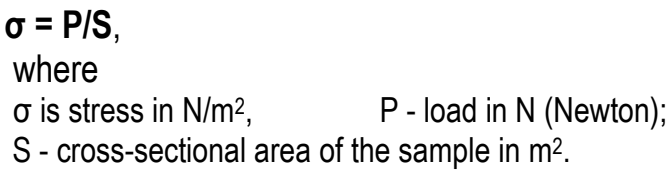

Thickness of the skin was determined nearerest to $0.01 \mathrm{~mm}$ using a microscope MP-2 at 5 points along the sample, and the average calculated.

\section{Isolation of skin type I collagen}

Type I collagen was extracted and isolated as described by Garbuzenko et al., (1997). Concentration of collagen was calculated as hydroxyproline content according to Stegmann and Staeder (1967) as modified by Utevskaya and Persky (1982).

\section{Analytical Determination of the Concentration of de novo Synthesized Collagen}

Determination of the concentration of de novo synthesized collagen in extracts was carried out as hydroxyproline using the method of Stegmann and Staeder (1967) as modified by Utevskaya and Persky, 1982).

\section{Analytical Determination of Free $\varepsilon$ - $\mathrm{NH}_{2}$-groups of Lysine and Hydroxylysine \\ Degree of oxidative deamination of lysine and hydroxylysine was evaluated as the content of free $\varepsilon$ - amino $\left(\varepsilon-\mathrm{NH}_{2}\right)$ and aldehyde $(\mathrm{COH})$ groups, which were measured using the methods of Kot et al. (2004) and Mazurov (1974), respectively. The methods used herein were based on getting and further identification of dinitro-flour-benzol-derivatives in the sum content of $\varepsilon$ - $\mathrm{NH}_{2}$-groups of lysine and hydroxylysine, arginine, asparagine and glutamine in proteins (Dubinina, 2000). The content of free $\varepsilon-\mathrm{NH}_{2}-$ groups of lysine and hydroxylysine was calculated as the difference between the sum total concentration of measured free $\varepsilon-\mathrm{NH}_{2}$-groups and free $\varepsilon-\mathrm{NH}_{2}$ groups of arginine.}

\section{Analytical Determination of Free Aldehyde Groups of Allysine and Hydroxyallysine}

Content of free aldehyde groups of allysine and hydroxyallysine in type 1 collagen was determined as described by Abraham et al (1982); Jin et al (2001).

\section{Preparation of collagen fibrils}

Fibrils $(0.2-0.3 \%)$ were prepared through their selfassembly (Persky et al., 2006) in type I collagen solution. Both type I collagen (in solutions, pH 7.0) synthesized without mechanical stretching and the one under the influence of mechanical stretching were allowed to stand for 3,10 and 22 days at $20^{\circ} \mathrm{C}$ 
so as to allow spontaneous formation of fibrils. The presence of fibrils in solution was monitored_using polarizing microscope MP-2 at 510-x magnification, and a digital camera (Canon A410 model).

\section{Histological examination of skin Samples}

After incubation of skin samples, $5 \times 5 \mathrm{~mm}$ of their fragments were cut out and immersed in $10 \%$ formalin for 24 hours for histological examination. After fixation, samples were washed in running water for 4 hours and then gradiently dried by $70 \%, 80 \%$, $96 \%, 100 \%$ ethyl alcohol for 24 hours in each alcohol. They were kept in a mixture of ethanolxylene and then in pure xylene for 2 hours and for a further 2 hours at $52{ }^{\circ} \mathrm{C}$. The samples were then placed in a mixture of xylene-paraffin and liquid paraffin. After cooling and solidification of paraffin, blocks were cut out and attached to wooden cubes. Sections were placed, after their unfolding, on glass slides. Saturated solution of picric acid in fuchsin was used in staining slides with sections. The preparations were again dried (drawing on battery with increasing alcohol concentration). Carboxylene and xylene were used to remove excess stains. Collagen fibers were stained in bright red colour.

\section{Measurement of thermodynamic parameters of denaturation of type I collagen}

Investigation of thermal stability of collagen fibrils was carried out at the rate of heating and concentration of collagen $10 \mathrm{sec} / \mathrm{min}$ in $1.4-2.3 \times 10$ ${ }^{5}$ cuvetes. Heat absorption curves were determined by melting point and enthalpy of denaturation obtained by measuring at microcalorimeter DASM- 4 (Garbuzenko et al., 1997).

Enthalpy of denaturation of collagen $(\Delta \mathrm{H})$ was calculated using the formula:

$$
\Delta \mathrm{H}=\frac{\mathrm{Pk}(\mathrm{T} 2-\mathrm{T} 1) \times \mathrm{S}}{\mathrm{Sk} \times \mathrm{V} \times \mathrm{m}_{\mathrm{p}}},
$$

where:
Pk - gauge power $=25 \mathrm{~h} 10^{-6}$ Tues

$\mathrm{T} 1$ and T2 are the temperature gauge on and off the power ' $\mathrm{K}$ '

$\mathrm{S}$ - area of the melting peak, $\mathrm{m}^{2}$

Sk- the area of the calibration stage, $\mathrm{m}^{2}$

$\mathrm{V}$ - the speed of heating, $\mathrm{A} / \mathrm{h}$

$m_{p}$ - mass of the protein in solution in the cell in grams

The values of $T 1, T 2$, and Sk were determined from a calibration curve, the value of ' $S$ ' - to the experimentally obtained melting curves.

Calculation of the entropy of denaturation was carried out as follows:

$\Delta S=\Delta \mathrm{N} / \mathrm{T}$, where:

$\Delta N$ - enthalpy of denaturation (DJ/g)

$T$ - temperature denaturation $(\mathrm{K})$

Heat absorption curves were determined by melting point and enthalpy of denaturation (Figure 1).

\section{Statistical Analyses}

Statistical analyses of the results were carried out using Student and Mann-Whitney criteria of reliability of test, using the software package 'Origin Pro 7.5 (Andronikashvily et al., 1972). Results with $p<0.05$ were considered significant.

\section{RESULT}

Table 1 shows the number of free $\varepsilon-\mathrm{NH}_{2}$-groups of lysine and hydroxylysine in collagen and indicated that, the free $\varepsilon-\mathrm{NH}_{2}$-groups increases with increasing tensile stress in the skin; free aldehyde groups, formed from the $\varepsilon-\mathrm{NH}_{2}$-groups of allysine and hydroxyallysine decrease. Table 2 shows the changes in solubility of Type 1 collagen with increase in force to indices mechanical stretching. Figure 1 compares the thermal stability of Type 1 collagens forms with and without tensile mechanical stress and table 3 show their thermodynamics parameters. Figure 3 shows the microphotographs of type I collagen fibrils formed in presence and absent of mechanical stress. Figure 4 is indicating the distribution by lengths of the fibrils formed from type I collagen synthesized in the skin in the absence of tensile mechanical stress, while Figure 5 shows the distribution by diameter.

Table 1: Effect of mechanical stretching of the skin on the content (mg/g of collagen) of free $\varepsilon-\mathrm{NH}_{2}$ - groups of lysine and hydroxylysine and aldehyde groups of allysine and hydroxyallysine in type I collagen,

\begin{tabular}{lllll}
\hline Free groups & \multicolumn{4}{c}{$\sigma, \mathbf{M N} / \mathbf{m}^{2}$} \\
\cline { 2 - 5 } & $\mathbf{0 . 0 0}$ & $\mathbf{0 . 0 7 5}$ & $\mathbf{0 . 1 2}$ & $\mathbf{0 . 2 4}$ \\
\hline $\boldsymbol{\varepsilon}-\mathrm{NH}_{2-}$ & $5.0 \pm 1.8$ & $32.0 \pm 2.0^{*}$ & $34.0 \pm 1.5^{*}$ & $28.0 \pm 3.2^{*, * *}$ \\
$\mathrm{COH}-$ & $52.0 \pm 3.1$ & $28.1 \pm 4.4^{*}$ & $26.0 \pm 2.9^{*}$ & $34.1 \pm 1.7^{*, * *}$ \\
\hline Note: ${ }^{*}$ - reliable $(\mathrm{p}<0.05)$ in relation with control $(\sigma=0) ;{ }^{* *}$ - reliable $(\mathrm{p}<0.05)$ in relation with former value of mechanical \\
stretching.
\end{tabular}


Table 2: Solubility of Type I exposed to mechanical stretching due to varying forces

\begin{tabular}{llccc}
\hline $\boldsymbol{\sigma}, \mathrm{MN} / \mathrm{m}^{2}$ & $\mathbf{0}$ & $\mathbf{0 , 1}$ & $\mathbf{0 , 1 8}$ & $\mathbf{0 , 2 4}$ \\
Solubility, \% & $1.50 \pm 0.18$ & $2.30 \pm 0.10^{*}$ & $2.60 \pm 0.20^{*}$ & $2.50 \pm 0.10^{*}$ \\
\hline
\end{tabular}

Note: * - reliable $(p<0,05)$ in relation with control $(\sigma=0)$

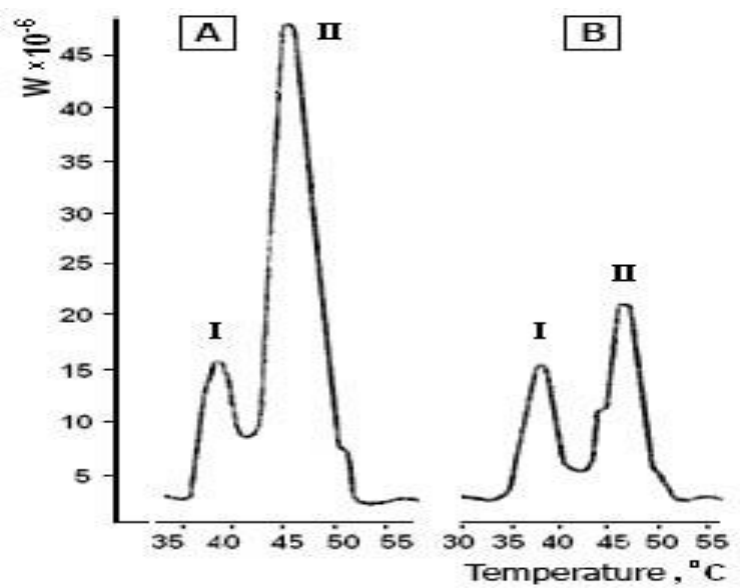

Figure 1: Heat absorption of fibrils formed from type I collagen, synthesized in the skin in the absence of tensile mechanical stress $A$ - under the effect of mechanical stretch of $0.18 \mathrm{MN} / \mathrm{m}^{2} \mathrm{~B}$ - after 10 days of incubation.

Table 3. Temperature $\left(T_{D}\right)$, enthalpy $(\Delta H)$ and entropy $(\Delta S)$ of denaturation of fibrils formed from type I collagen synthesized in the skin in the absence of tensile mechanical stretching and under the effect of mechanical stretching of $0.18 \mathrm{MN} / \mathrm{m}^{2}$, after 10 days of incubation

\begin{tabular}{ccc|cc}
\hline \multirow{2}{*}{ Index } & $\begin{array}{c}\boldsymbol{\sigma}=\mathbf{0} \\
\text { Melting }\end{array}$ & $\begin{array}{c}\text { Melting } \\
\text { point I }\end{array}$ & $\begin{array}{c}\boldsymbol{\sigma}=\mathbf{0 . 1 8 ~} \mathbf{M N} / \mathbf{m}^{2} \\
\text { Melting } \\
\text { point I I }\end{array}$ & $\begin{array}{c}\text { Melting } \\
\text { point II }\end{array}$ \\
\hline $\mathrm{T}_{\mathrm{D}},{ }^{0} \mathrm{C}$ & $38.6 \pm 0.4$ & $46.0 \pm 0.3$ & $38.0 \pm 0.2$ & $45.3 \pm 0.4$ \\
$\Delta \mathrm{H}, \mathrm{Dj} / \mathrm{g}$ & $78.8 \pm 2.6$ & $133.0 \pm 5.9$ & $78.8 \pm 1.6$ & $90.9 \pm 3.1^{*}$ \\
$\Delta \mathbf{S}, \mathrm{Dj} / \mathrm{g} \times$ degree & $0.25 \pm 0.03$ & $0.42 \pm 0.03$ & $0.25 \pm 0.04$ & $0.28 \pm 0.06^{*}$ \\
\hline
\end{tabular}

Note. ${ }^{*}$ - reliable $(p<0,05)$ with relation to control $(\sigma=0)$
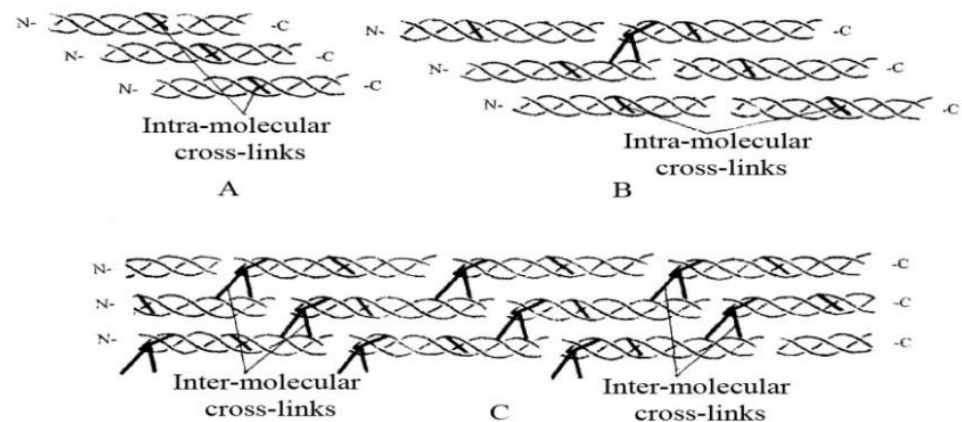

Figure 2. A schematic representation of type I collagen based on the degree of inter-molecular cross-linking: A - a short fibril in which molecules in adjacent rows are bound only by electrostatic and hydrophobic interactions. B medium-size fibril with small number of inter-molecular cross-links. $\mathrm{C}-$ a long fibril, in which molecules in adjacent rows are bound by inter-molecular cross-links and less by electrostatic and hydrophobic interactions. Intramolecular cross-linking does not affect the length of fibrils. 


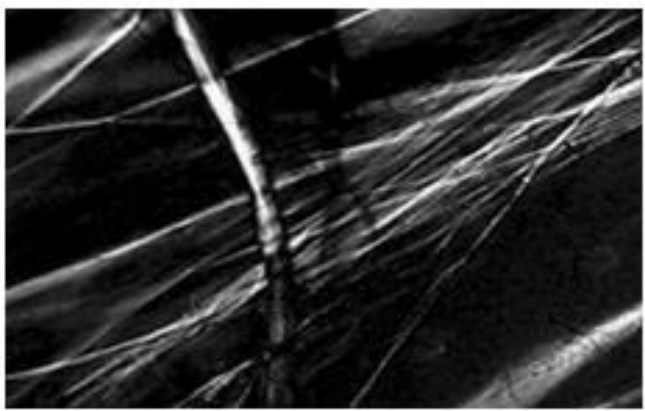

A

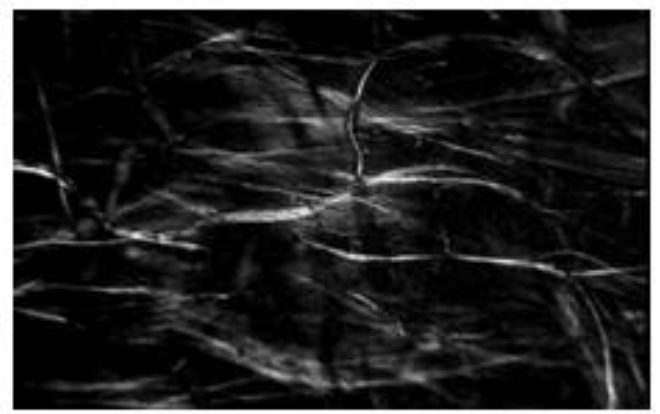

Б

Figure 3. Microphotographs of fibrils formed from type I collagen synthesized in the skin in the absence of tensile mechanical stretching (A), and under the stress of $0.18 \mathrm{MN} / \mathrm{m}^{2}(B)$ after 10 days of incubation.
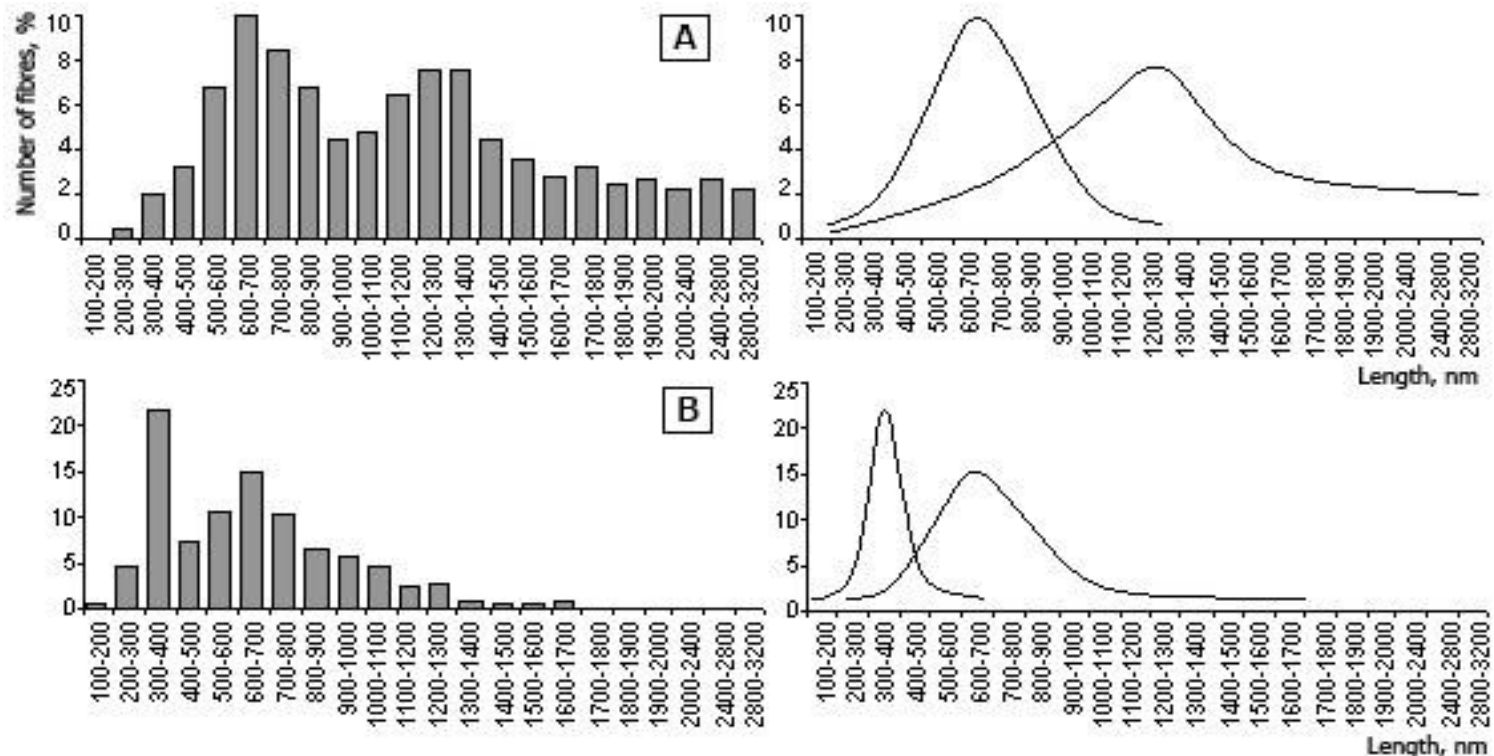

Figure 4. Distribution by lengths of the fibrils formed from collagen type I synthesized in the skin in the absence of tensile mechanical stress $(A)$, under the influence of mechanical stretching of $0.18 \mathrm{MN} / \mathrm{m}^{2}(B)$, after 10 days of incubation.
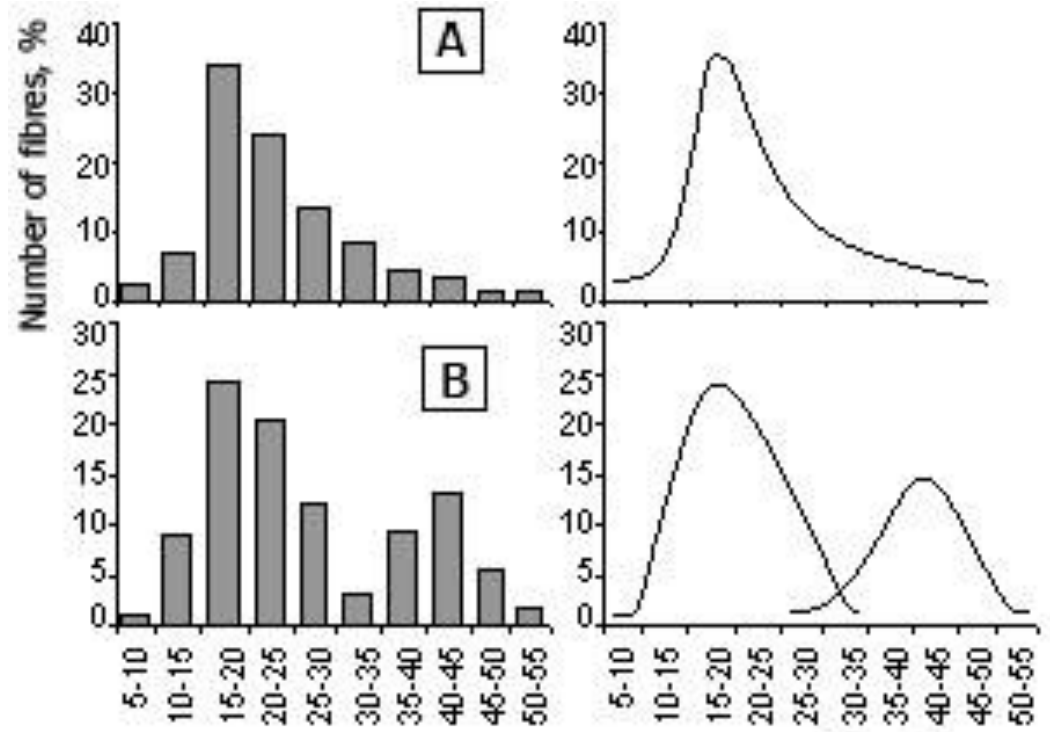

Diameter, $\mathrm{nm}$

Figure 5. Distribution by diameter, of fibrils formed from type I collagen, synthesized in the skin in the absence of tensile mechanical stretching (A), and at $\sigma=0.18 \mathrm{MN} / \mathrm{m}^{2}$ (B), after 10 days of incubation. 


\section{DISCUSSION}

The observed increase in free $\varepsilon-\mathrm{NH}_{2}$-groups with increasing tensile stress on the skin, with decreased free aldehyde groups, formed from the $\varepsilon-\mathrm{NH}_{2}$-groups of allysine and hydroxyallysine could be the result of decreased activity of lysineoxydase (Table 1). The mechanism of this phenomenon may be, due to a sharp increase in the concentration of collagen as a result of intensification of its synthesis which does not keep in phase with the prevailing enzyme concentration.

Free aldehyde groups are intermediate products of cross-covalent bonds (Fessler, 2004). The observed decrease in the number of these groups in the collagen synthesized under mechanical stress lowers the degree of cross-covalent binding, as evidenced by reduced solubility with increasing mechanical stretching (Table 2).

From Figure 1, the curves of the dependence of heat absorption on temperature for fibrils formed from type I collagen, synthesized in the skin in the absence of tensile mechanical stress and under the influence of a strain of $0.18 \mathrm{MN} / \mathrm{m}^{2}$, and incubated for 10 days at $18{ }^{\circ} \mathrm{C}$ are shown. In both cases, the relationships are characterized by two peaks; and the heights and peak areas for each of the curves are different. This may indicate the heterogeneity of all studied samples which consist of at least two fractions probably relative to force of stress. Secondly, the relative contents of these fractions in the samples of fibrils formed from collagen, synthesized in the skin in the absence and under the effect of mechanical stretching are different. Thirdly, the energy required to destroy fibrils that belong to peaks I and II, varies. The corresponding values of entropy and enthalpy of melting points of the fibrils are shown in Table 3. The results may implies that type I collagen synthesized in the absence of mechanical stress is a molecule of a rigid rod-shaped triple helix of polypeptide chains with a diameter and length of about 0.15 and $300 \mathrm{~nm}$, respectively. In accordance with a specific primary structure of polypeptide chains along the surface of the molecule, areas consisting of hydrophobic and polar amino acid residues alternate. This distribution of radical amino acid residues along the surface of the molecule is the basis of the pronounced ability of type I collagen to self-assembly in the sub-molecular structures, i.e., in fibrils (Persky et al., 2006). Aggregation takes place in the lateral direction due to the interaction of hydrophobic and polar regions with adjacent molecules that are arranged in a parallel manner, and shifted relative to each other by a quarter of their length. In the longitudinal direction, fibrils grow by forming inter-molecular bonds between $\varepsilon-\mathrm{NH}_{2}$-groups of lysine and hydroxylysine, and $\mathrm{COH}$-groups of allysine and hydroxyallysine in the $\mathrm{N}$ - and $\mathrm{C}$-terminal regions of collagen molecules arranged alternatively and located in adjacent rows.

Structure of fibrils and the influence of the degree of inter-molecular cross-linking on them are shown in Figure 2. A large number of calorimetric studies (Abraham et al., 1975) showed that, denaturation curves of freshly isolated from tissues, collagen molecules, have the same melting point. Freshly isolated type I collagen synthesized in the absence and presence of mechanical stretching is also characterized by a melting point (Stepanova et al., 1996).

Therefore, the observed two-stage denaturation of fibrils may indicates that, formation of fibrils in solution at a temperature and $\mathrm{pH}$ near the physiological condition, is not accompanied by a thermal effect. At the same, the melting point of fibrils formed under these conditions is about $80 \mathrm{DJ} / \mathrm{g}$ of protein. This value coincides with heat of denaturation of individual type I collagen molecules from rats' skin, and is close to the peak values of the collagen synthesized in both absence and presence of mechanical stretching of the skin (Table 3). This coincidence may indicates that, the first stages of fibril formation are determined mainly by weak hydrophobic and electrostatic interactions of complementary sites present in polar and non-polar regions of collagen molecules. These interactions apparently do not contribute significantly to the total structural energy of the formed aggregates. Therefore, peak I apparently corresponds with that of mixture of aggregates that fully contain, or contain less number of inter-molecular cross-links.

The emergence of strong high-energy covalent bonds between collagen molecules in fibrils, as a result of aldol condensation and the formation of Schiff's bases, takes place only after some time, i.e., after the formation of primary aggregates (Grazi et al., 1963). In accordance with our findings, at least 10 days are required for fibrils to sufficiently form such bonds so that, the energy of their destruction is much higher than the energy of denaturation of fibrils that are only bound through electrostatic and hydrophobic interactions. Therefore, peak II represents heat absorption of fibrils that contain large number of inter-molecular cross-links. 
However, a much smaller value of the enthalpy of denaturation of fibrils that are related to this peak, and are formed from collagen (Table 3, peak II) under mechanical stress indicates that, they contain a smaller number of inter-molecular cross-links, compared with collagen synthesized in the absence of stress. This is a direct consequence of a decrease in oxidative deamination of $\varepsilon$ - $\mathrm{NH}_{2}$-groups of lysine and hydroxylysine in type I collagen, which is synthesized in the skin under the effect of mechanical stretching (Table 1).

Different numbers of inter-molecular cross-links in fibrils must lead to differences in their structure (Jin et al., 2001). Micrographs of fibrils formed from type I collagen synthesized in the skin in the absence of tensile mechanical stretching, and under the effect of $0.18 \mathrm{MN} / \mathrm{m}^{2}$ strain, incubated for 10 days at $20{ }^{\circ} \mathrm{C}$, are shown in Figure 3 , and distributions of both types of fibrils by length and diameter are represented in Figures 4 and 5 . It could be seen from the microphotographs (Figure 3 ) that the morphology of fibrils formed from type I collagen synthesized in the absence of, and under the influence of mechanical stretching in the skin are different. While the lengths of fibrils in the first lie in a wide range, from 200 to $3200 \mathrm{~nm}$ with a maximum of $600-700 \mathrm{~nm}$, corresponding to twice the length of the molecules of type I collagen, their lengths in the second microphotograph do not exceed $1700 \mathrm{~nm}$, with a maximum number of fibres of $300-400 \mathrm{~nm}$ long, i.e. the length of a single molecule. Characteristically, the maximum number of these fibers is $13 \%$ higher than the maximum number of fibres of the first type in ' $A$ '. Therefore, during the process of temporary development, fibrils formed from type I collagen synthesized in the skin in the absence of mechanical stretching averagely become longer than those which are formed from collagen synthesized under the influence of stretching. The diameters of fibrils in both cases are within the range of 5 to $55 \mathrm{~nm}$ (Figure 5). In this case, fibrils formed from collagen synthesized under the influence of mechanical stretching have two values of diameter - $15-25 \mathrm{~nm}$ and $35-45 \mathrm{~nm}$, while those fibrils formed from collagen, synthesized in the skin in the absence of mechanical stretching, have only one - at $15-20 \mathrm{~nm}$.

\section{CONCLUSION}

In conclusion, it is therefore, assumed that, the observed decrease in the degree of oxidative deamination of $\varepsilon-\mathrm{NH}_{2}$-groups of lysine and hydroxylysine in type I collagen synthesized in the skin under the influence of mechanical stress, leads to disruption of the formation of fibrillar collagen structures that also leads to decrease in the thermal stability of fibrils that are formed from collagen structures.

\section{Acknowledgements}

Authors of this article are hereby grateful to the Heads, Department of Human and Animal Physiology, and that of Biochemistry of the V. N. Karazin Kharkov National University, Ukraine, for advice, and assistance.

\section{REFERENCES}

Abraham, P.A., Perejda, A.J., Cames, W.H. and Uitto, J. (1982). Marfan syndrome Demonstration of abnormal elastin in aorta. The Journal of Clinical Investigation, 70(6):1245-52.

Abraham, P.A., Smith, D.W. and Cames, W. H. (1975). Biosynthesis of lysyn-derived elastin cross-linking in aortic cell cultures. Biochemical and Biophysical Research Communications, 67(2): 723-727.

Andronikashvily, Ye. L., Monaselidtse, D.R., Bakradze, N.G. and Mrevlishvily, G.M. (1972). Plotting of Diagram on the Condition of Procollagen Solutions. Molecular Biology, 6(6):. 915 - 925. Article in Russian language.

Buschmann, M.D., Gluzband, Y.A., Grodzinsky, A.J. and Hunziker, E.B. (1995). Mechanical compression modulates matrix biosynthesis in chondrocyte/agarose culture. Journal of Cell Science, 108: 1497-1508.

Dubinina, E.E. (2000). Oxidative modification of blood plasma proteins in patients with psychiatric disorders. Questions on Medicinal Chemistry, 4: 36-47. Article in Russian Language.

European Convention (1985). On conservation of vertebrate animals used in experiments and other scientific aims Available from: Cовem Европы. - Протокол ЕTS №170. Страсбуре.

Eyre, D.R. and Wu, J-J. (2005). Collagen CrossLinks. Topics in Current Chemistry, 247: 207229.

Fessler, J.H. (2004). Self-assembly of collagen. Journal of Supramolecular Structure, 2(2-4): 99 $-102$.

Garbuzenko, O. B., Emeth, Ye. B and Persky, E.E. (1997). The in vitro effect of deformation on protein metabolism and mechanical properties of rats' skin and aorta. Bulletin of Biology and Medicinal Problems, № 25. P.12-18. Article in Russian language. 
Grazi, E., Meloch, H., Martinez, G., Wood, W and Horecker, B. L. (1963). Evidence for Schiff base formation in enzymatic aldol condensations. Biochemical and Biophysical Research Communications, 10(1):4-10.

Greene, S. A. (2002). Veterinary anesthesia and pain management secrets.- Henlay \& Belfuc Inc, P.4646; 266 .

Hyun, J., Chen, J., Setton, L and Chilkoti, A. (2006). Patterning cells in highly deformable microstructures: Effect of plastic deformation of substrate on cellular phenotype and gene expression. Biomaterials, 27(8): 1444-1451.

Jin, M., Frank, E.H., Quinn, T.M., Hunziker, E.B. and Grodzinsky, A.J. (2001). Tissue Shear Deformation Stimulates Proteoglycan and Protein Biosynthesis in Bovine Cartilage Explants. Archives of Biochemistry and Biophysics, 395(1): 41 - 48.

Kot, Yu. G., Zdjukova, T. V., Deng, Bo and Persky, E. E. (2004). Hydroxylation of Proline in Skin and Aorta Collagen Under in vitro Action of Mechanical Stress on them. Current Problems in Biology and Medicine, 1: 269-272. Article in Russian language.

Mazurov, V. I. (1974). Biochemistry of collagenous proteins. Moscow: Medicine. Book in Russian language. pp. 248.
Persky, E.E., Nikitina, N.A., Naglov, A.V. and Kot, Yu.G. (2006). Age-related characteristic features of the induction of synthesis and intensity of some stages of collagen processing in the connective tissue under the effect of mechanical loading Biological Bulletin of V. N. Karazin Kharkov National University, Ukraine, 2. Vol. 10. P. 126-129. Article in Russian language.

Stegmann, H and Staeder, K. (1967). Determination of Hydroxyproline. C. Chin Edn. Chim. Publ. 18(2): 267-273.

Stepanova, O. V., Biryukov, K. G., Shirinsky, V. N and Tkachuk, V. A. (1996). Effect of periodic stretching of smooth muscle cells on the expression of marker proteins of contractile phenotype. Physiology Journal, 82(9): 16-27. Article in Russian language.

Sun Y., Chen W.-L., Lin S.-J., Jee S. H, Chen Y.-F, Lin L. C., So, P.T.C., Dong, C. Y. (2006). Investigating Mechanisms of Collagen Thermal Denaturation by High Resolution SecondHarmonic Generation Imaging. Biophysical Journal, 91(7): 2620-2625.

Utevskaya, L. A and Persky, E. E. (1982). Simple Method of Determining Free and Total Hydroxyproline. Bulletin of Kharkov University, USSR. 226: 18-20. Article in Russian language. 